\title{
Organisations paysannes et développement socio économique dans la zone forestière du Cameroun méridional, 1992 à 2015
}

\author{
Organizações camponesas e desenvolvimento socioeconômico na \\ zona florestal do sul dos Camarões, 1992 a 2015
Rural organisations and socioeconomic development in the forest zone of south Cameroon, 1992-2015

Christophe Signie*

Resumo: O objetivo deste artigo é mostrar o contributo das organizações camponesas para a promoção do mundo rural na zona florestal do sul dos Camarões desde 1992, ano em que um decreto do presidente da República autorizou a criação de grupos de agricultores, até 2015. Com base em fontes arquivísticas, fontes orais e uma bibliografia seletiva, este artigo trata do surgimento das organizações de agricultores nas regiões do Camarões Central e do Sul e aborda as suas atividades e o seu impacto ao desenvolvimento rural. Nossas análises mostram que a retirada do Estado do setor produtivo na década de 1990 e o quadro legislativo implementado pelas autoridades públicas favoreceram a criação de várias organizações no mundo rural na zona florestal. Essas associações camponesas contribuem para o desenvolvimento rural através do desenvolvimento da agricultura, do processamento e da comercialização de produtos e de fundos de poupança. $\mathrm{O}$ dinheiro dessas atividades permite que os membros dessas associações camponesas melhorem suas condições de vida.

Palavras-chave: Organizações camponesas, Desenvolvimento, Zona florestal, Camarões

Résumé: L'objet de cet article est de montrer la contribution des organisations paysannes à la promotion du monde rural dans la zone forestière du Cameroun

(c) EY Direito autoral e licença de uso: Este artigo está licenciado sob uma Licença Creative Commons. Com essa licença você pode compartilhar, adaptar, para qualquer fim, desde que atribua a autoria da obra, forneça um link para a licença, e indicar se foram feitas alterações. 
méridional de 1992, année au cours de laquelle un décret du président de la république autorisait la création des regroupements paysans à 2015. Quels sont les facteurs d'émergences des organisations paysannes dans les régions du Centre et du Sud Cameroun? Quels sont leurs activités et leur impact sur le développement de la zone étudiée ? A partir des sources d'archives, des sources orales et d'une bibliographie sélective, cet article apporte des réponses à ces questions. Il ressort de nos analyses que le désengagement de l'Etat du secteur productif dans les années 1990 et le cadre législatif mis en place par les pouvoirs publics ont favorisé la création de plusieurs GIC dans le monde rural dans la zone forestière. Ces associations paysannes contribuent à la promotion du monde rural à travers le développement de l'agriculture, la transformation et la commercialisation des produits ainsi que l'épargne. L'argent issu de ces activités permet aux planteurs membres de ces associations paysannes d'améliorer leurs conditions de vie.

Mots clés : Organisations paysannes-Développement-Cameroun-Zone forestière

\begin{abstract}
Abstrat: The purpose of this article is to evaluate the contribution of rural organisations to the socio economic development in the forest zone of the south from 1992; the year a Presidential decree authorised the creation of common initiative groups to 2015 . This aim is realised with respect to the following research questions: What factors led to the emergence of common initiative organisations in the Centre and Southern Regions of Cameroon? What were their activities and the impact of the groups in the areas under study? The answers to these questions benefited from the following sources: archival, oral and a selective bibliography. Our analysis reveals that the disengagement of the government from the agricultural sector in the 90s and the regulations enforced by stakeholders favoured the creation of so many common initiative groups in the forest zone of Cameroon. The article concludes that the common initiative organisations largely contributed to rural development in Cameroon. This was through the transformation of agricultural products, its commercialisation and the saving of money by the members of the farmer groups that also permitted them to improve on their living standards.
\end{abstract}

Keywords: Common initiative organisations, Development, Cameroon, forest zone

\title{
Introduction
}

En Afrique en général et au Cameroun en particulier, les organismes d'intervention en milieu rural à savoir les coopératives et les sociétés de 
développement ont été pendant longtemps créés par l'Etat. Ces structures accompagnaient les paysans dans l'accomplissement de leurs travaux à savoir la production, la transformation et la commercialisation de leurs produits. Mais à partir de 1990, la crise économique aidant, l'Etat s'est retiré du secteur productif, entraînant de ce fait la faillite de la plupart de ces structures. Pour combler le vide, les paysans et certains particuliers se sont regroupés pour créer ce qu'on appelle les organisations paysannes. Pour Georges Courade, une organisation paysanne est le lieu d'élaboration des stratégies collectives pour tenir compte de la multiplicité des logiques paysannes et valoriser les structures communautaires villageoises, les solidarités existantes, les associations entre villageois. ${ }^{1}$ Le terme développement désigne l'ensemble des modifications positives intervenues dans les conditions matérielles de l'existence des populations, qui les a fait passer de la misère originelle à la prospérité relative, de sorte qu'elles vivent mieux qu'auparavant. ${ }^{2} \mathrm{La}$ préoccupation des auteurs qui se sont intéressés à l'étude des organisations paysannes est différente de la notre. C'est ainsi que par exemple dans son mémoire de fin de formation, P. Menye Atangana ${ }^{3}$ ne s'intéresse qu'aux activités des regroupements paysans dans la Lékié. Cette étude a pour lacune de se cantonner dans un seul département et n'aborde pas l'impact de ces organisations sur le développement. Dans son mémoire d'économie et de sociologie rurale, Esther Ekoué Ekoué dresse la typologie des organisations paysannes en zone rurale, leurs principales caractéristiques et les problèmes auxquels elles sont confrontées ${ }^{4}$. L'absence de la méthode diachronique propre aux historiens ne permet pas de mieux appréhender l'impact de ces structures sur le développement. C'est pourquoi, nous avons jugé utile d'écrire cet article pour montrer l'impact des organisations paysannes dans le développement de la région forestière du Cameroun méridionale. Cette étude répond aux questions suivantes : Quels sont les facteurs d'émergences des organisations paysannes dans les régions du Centre et du Sud Cameroun? Quels sont leurs activités et leur impact sur le développement de la zone étudiée?

A partir des sources orales, des sources d'archives et d'une bibliographie sélective, cette étude analyse les facteurs de création des structures paysannes et étudie leur contribution au développement économique de la zone étudiée.

\section{Les facteurs d'émergence des organisations paysannes}

Plusieurs facteurs ont concouru à la création des organisations paysannes au Cameroun et parmi eux, le désengagement de l'Etat du secteur productif

\section{Le désengagement de l'Etat du secteur productif}

Avant la libéralisation et par le biais de divers mécanismes (projets de développement, sociétés d'Etat et recours à la subvention,), l'Etat assurait de nombreux services aux producteurs agricoles et aux éleveurs : fournitures des 
intrants, services de soins de santé animale, production de semences, plants et autres matériels biologiques, appui à la commercialisation. Cette époque de vache grasse est finie depuis 1990. Les réformes mises en œuvre ont porté sur la suppression des subventions, la libéralisation du commerce des engrais, des pesticides, des produits et matériels vétérinaires et des provendes. Les services de santé animale ont été privatisés et une législation phytosanitaire promulguée. L'Etat s'est retiré de la plupart de ces fonctions en faisant l'hypothèse que le secteur privé prendrait vite le relais. ${ }^{5}$

Plusieurs raisons ont amené les pouvoirs publics à se désengager du secteur productif. En 1992 en effet, le contexte économique camerounais est marqué par la transition entre les plans quinquennaux et les programmes d'ajustement structurel. Si les plans quinquennaux sont l'ensemble des mesures gouvernementales pour planifier l'activité économique, les programmes d'ajustement structurel sont la réponse des autorités publiques à la crise économique constatée en 1980. Cette période de soudure a été très difficile pour l'Etat camerounais qui contrôlait l'essentiel des investissements dans le domaine agricole et régulait son dynamisme par un système de prix dont il maîtrisait seul les contours. ${ }^{6}$

En 1990, une politique agricole a été élaborée pour améliorer les performances. Ses objectifs comprennent entre autre la modernisation de l'appareil de production, la sécurité alimentaire, la promotion et la diversification des exportations, le développement de la transformation des produits agricoles et l'équilibre des filières de production.

Ainsi, les raisons qui amènent l'Etat à se désengager du secteur productif sont l'environnement économique difficile et les mesures qu'il prend lui-même pour trouver un équilibre. Dans ce contexte, les pouvoirs publics sont à bout de souffle et se retrouvent dans l'incapacité d'assumer les fonctions qui étaient les siens sur le plan agricole. C'est pourquoi, l'Etat s'est désengagé de la sphère de production en facilitant les activités des opérateurs privés et en donnant par la même occasion une énorme responsabilité aux paysans, principaux producteurs. Un autre facteur ayant favorisé la création des regroupements paysans au Cameroun concerne le cadre législatif.

\section{Le cadre législatif régissant les organisations paysannes}

La libéralisation, en supprimant les mécanismes de régulation administrée, a laissée face à face des opérateurs économiques le plus souvent peu préparés à s'engager sur de nouveaux types de relations basées sur la négociation et l'établissement des liens contractuels. La situation des producteurs agricoles s'est aggravée par des rapports déséquilibrés avec les autres opérateurs économiques, entre d'un côté, une offre le plus souvent atomisée et de l'autre, des opérateurs au degré d'intégration et de concentration développée comme les oligopsones de firmes multinationales, les commerçants collecteurs, les grossistes et bien d'autres. Pour rééquilibrer le pouvoir de 
négociation en faveur des groupes les plus vulnérables, le gouvernement appuie la promotion des organisations professionnelles agricole. ${ }^{7}$

C'est pourquoi, dans le cadre de la mise en œuvre de la politique de libéralisation de l'économie et de l'application des orientations des nouvelles politiques agricoles, forestières et environnementales, l'Etat a mis en place dès 1990 une nouvelle réglementation favorisant la création des organisations paysannes et communautaires. Il s'agit de la loi numéro 90/053 du 19 décembre 1990 sur la liberté d'association. Ce texte remplace la loi nº7/LF/19 du 12 juin 1967 qui elle-même abrogeait la loi de 1901. Cette loi s'est avérée plus souple et donne le pouvoir aux préfets de certifier l'existence des associations. ${ }^{8}$

Par la suite, la loi n92/006 du 14 août 1992 concernant les sociétés coopératives et les groupes d'initiatives communes fut promulguée, en remplacement de la loi $\mathrm{n}^{\circ} 73 / 10$ du 07 décembre 1973 portant statut des sociétés coopératives. A travers cette loi, de nombreuses organisations de bases, ayant un nombre de membres réduit, ont été légalisées et se sont engagées dans un processus de structuration en unions et fédérations de groupes d'initiatives communes. Cette loi a également permis aux producteurs agricoles, en particulier dans les filières café et cacao, de se libérer d'un long passé coopératif dirigiste où la non-transparence dans la gestion a créé des attitudes de rejet du terme coopérative chez les agriculteurs. C'est dans le sillage de la loi de 1992 que le registre des coopératives et GIC a été créé pour permettre parmi d'autres attributions de légaliser ces formes d'organisations paysannes. Il faut toutefois relever que la souplesse de cette loi a laissé la place à des dérives. Des sociétés privées de prestations de services, n'ayant rien à voir avec les activités agricoles ont pu se légaliser en GIC pour échapper à la fiscalité. ${ }^{9}$

Après la promulgation de ces deux premières lois, d'autres suivirent. Il s'agit de la loi de 1993 sur les groupements d'intérêts économique, de la loi n⿳94/01 du 20 janvier 1994 portant régime des forêts, de la faune et de la pêche et ses décrets d'application, et enfin de la loi cadre relative à la gestion de l'environnement. C'est tout cet arsenal législatif qui régit les organisations paysannes au Cameroun. Il a permis l'essor des regroupements paysans avec l'appui des programmes mis en place par l'Etat et l'aide des bailleurs de fonds internationaux. Parmi ces lois, celle sur les coopératives et les GICS est celle qui a été conçue pour les organisations de producteurs à vocation agricole. Toutefois, la loi n92/006 du 14 août 1992 prévoit deux principales formes juridiques pour les organisations à vocation agricole à savoir les GIC et les coopératives. La légalisation des GIC et des coopératives s'effectue auprès du service régional du registre des sociétés coopératives et des groupes d'initiative commune logés au sein de la délégation régionale du Ministère de l'agriculture et du développement rural. ${ }^{10} \mathrm{C}$ 'est grâce à cette législation que les organisations paysannes ont pu naître au Cameroun en général et dans la zone forestière en particulier. 


\section{L'Essor des organisations paysannes dans la zone forestière}

La zone forestière est l'une des régions du Cameroun où les premières organisations paysannes sont apparues après l'entrée en application de la loi du 14 août 1992 portant création des sociétés coopératives et des groupements d'initiatives communes. Ces structures formelles de travail collectif ont pour objectif d'aider leurs membres à mener à bien des activités de production, de transformation et de commercialisation des ressources économiques, de leur fournir une assistance matérielle et financière et de les aider à épargner de l'argent dont ils se servent ensuite pour se soigner, s'équiper, régler la scolarité de leurs enfants et pour faire face à des situations difficiles. ${ }^{11}$

En 2001, le Centre comptait 328 GICS ruraux en activités dont 119 groupements féminins, 77 groupements masculins et 132 groupements mixtes totalisant 8610 adhérents. 97 GICS sont implantés dans le département de la Lékié, 37 dans la Mefou et Afamba, 49 dans le Mbam et Kim, 41 dans le Nyong et So'o, 29 dans la Haute Sanaga, 30 dans le Mbam et Inoubou, 20 dans le Nyong et Mfoumou, 23 dans la Mefou et Akono et 17 dans le Nyong et Kelle. ${ }^{12}$

Ces organisations paysannes rassemblent chacune entre 15 et 20 personnes, hommes et femmes confondus. Elles sont gérées par trois organes à savoir l'Assemblée des membres, le comité directeur et le comité de contrôle. L'assemblée générale regroupe tous les membres de l'association et c'est elle qui exécute tous les travaux. Le comité directeur est composé des dirigeants de la structure. Mais dans certaines organisations, la structure dirigeante laisse apparaitre le président, le secrétaire, le trésorier, le caissier, le contrôleur, le conseiller, le chargé des travaux, le chef des chantiers, le mobilisateur, le chargé de la logistique, le cantinier et le chargé des relations publiques. ${ }^{13}$ C'est grâce à cette structure que les organisations paysannes se déploient sur le terrain.

\section{Les activités des organisations paysannes}

Les activités socioéconomiques des regroupements paysans en milieu rural dans la zone forestière du Cameroun méridional comprennent l'agriculture, la transformation et la commercialisation des produits, l'épargne et bien d'autres.

\section{L'agriculture}

La plupart des organisations paysannes dès leur création s'investissent tout d'abord dans l'agriculture. Elles sont motivées par le souci d'augmenter la production et d'accroître leurs fonds de caisse. Les activités commencent généralement par le choix du site, la création et l'exploitation des champs communautaires des cultures vivrières. L'acquisition des terres se fait soit par achat, soit par don à travers la mise à disposition par des notables ou des grandes familles des espaces à cultiver. Les superficies des champs varient entre 2,5 et 15 hectares. ${ }^{14}$ 
En vue de la production, les paysans préparent d'abord les champs. Cette préparation se fait à l'aide des tracteurs pour les organisations paysannes les plus nantis. Après les labours viennent les semailles. Cette activité déterminante dans la production agricole nécessite un investissement personnel de chaque membre. Dans le GIC Oyili Bininga de Mbélé par exemple, les tâches sont bien reparties entre les membres dans leur plantation de manioc. Un groupe de personnes dispose les boutures sur la terre labourée par les engins tandis qu'un autre s'occupe de les enfouir au sol. ${ }^{15}$

Dans les différents champs collectifs, les groupements d'initiative commune cultivent des denrées facilement consommables ou vendables dans les marchés locaux. Il s'agit de la banane plantain, du maïs, du manioc, des courges, du macabo, de la tomate, du piment et bien d'autres produits. ${ }^{16} \mathrm{~A}$ titre d'exemple, le GIC dénommé comité de développement de la vallée de Kya créé en 1992 dans le département de la Mefou et Afamba à Awae a produit sur un espace de 2700 hectares 4500 tonnes de plantain, $6000 \mathrm{t}$ de tubercules de manioc et $3100 \mathrm{t}$ de macabo. ${ }^{17}$ Au cours de la même année, le GIC Mat, créé en 1993 dans l'arrondissement de Mbangassina dans le département du Mbam et Kim a récolté $109 \mathrm{t}$ d'ananas, $25 \mathrm{t}$ de tomates, $14 \mathrm{t}$ de poivrons et $9 \mathrm{t}$ de piment. ${ }^{18}$ Le GIC Apeyl quant à lui produit depuis 2009 une gamme variée de culture vivrière. Le tableau 1 montre l'évolution de la production de ce GIC de 2009 à 2013.

Tableau 1. Evolution de la production vivrière du GIC Apeyl de 2009 à 2013

\begin{tabular}{c|c|c|c|c|c}
\hline \multirow{2}{*}{ Cultures } & \multicolumn{5}{|c}{ Années et productions } \\
\cline { 2 - 6 } & $\mathbf{2 0 0 9}$ & $\mathbf{2 0 1 0}$ & $\mathbf{2 0 1 1}$ & $\mathbf{2 0 1 2}$ & $\mathbf{2 0 1 3}$ \\
\hline Maïs & $3 \mathrm{t}$ & $4 \mathrm{t}$ & $5 \mathrm{t}$ & $8,7 \mathrm{t}$ & $9,5 \mathrm{t}$ \\
\hline Arachides & 6 sacs & 8 sacs & 9 sacs & 13 sacs & 15 sacs \\
\hline Manioc & 50 cuvettes & 65 cuvettes & 95 cuvettes & 185 cuvettes & 200 cuvettes \\
\hline Soja & 0 & 2 cuvettes & 3 cuvettes & 0 & 0 \\
\hline Oignons & 2 cuvettes & 3 cuvettes & 4,5 cuvettes & 0 & 0 \\
\hline
\end{tabular}

Source : Rapport d'activités du GIC Apeyl de 2009 à 2013

Il ressort de ce tableau que la principale culture vivrière pratiquée par les membres du GIC Apeyl est le maïs dont la production est allée croissante, passant de $3 \mathrm{t}$ en 2009 à $9,5 \mathrm{t}$ en 2013. Il en est de même du manioc avec près de 200 cuvettes récoltés en 2013 contre 50 cuvettes en 2009. Les arachides occupent aussi une place de choix avec 15 sacs produits en 2013. La priorité accordée à ces cultures provient du fait qu'elles sont facilement commercialisables et peuvent aussi être consommée dans les familles. 
Quant au GIC CEL dans la Lékié, il a produit en 2012 environ 4611 Kgs de maïs sur une superficie de 6,5 ha, 249 filets de $50 \mathrm{kgs}$ de patate sur 2 ha, 30 sacs de $50 \mathrm{kgs}$ d'arachides sur 7 ha, $100 \mathrm{kgs}$ de Soja sur $2500 \mathrm{~m}^{2}, 385$ paquets de légumes sur $600 \mathrm{~m}^{2}$ et 350 litres d'huile de palme sur 3 ha. ${ }^{19}$

En dehors de la production des champs communautaires, il existe aussi les productions individuelles des paysans, membres des GICS. A titre d'exemple, le tableau 2 montre les productions individuelles des membres du GIC Oliga.

Tableau 2. Production individuelle des membres du GIC Oliga

\begin{tabular}{c|c|c|c}
\hline Cultures & Productions & Superficies (ha) & Prix (FCFA) \\
\hline Macabo & 8 à 20 bacos & 1 & $2000 /$ baco \\
\hline Plantain & 3 à 4 régimes & 1 & Non déterminé \\
\hline Arachide & 3 à 5 sacs & 1 & $15000 /$ sacs de $100 \mathrm{kgs}$ \\
\hline Igname & 3 bacos & 0,5 & $15000 /$ baco \\
\hline Manioc & 4 bacos & 1 & $1500 /$ baco \\
\hline
\end{tabular}

Source :Ekoué Ekoué. "Contribution des organisations”, p.47.

Il ressort de ce tableau que les superficies des champs individuelles varient de 0,5 à 1 ha. Parmi les cultures pratiquées, la production du macabo est la plus importante. Les revenus des paysans proviennent de la vente de ces denrées qui sont récoltées deux à trois fois par an.

En dehors des cultures vivrières, les regroupements paysans pratiquent aussi les cultures de rentes. C'est ainsi que dans la Lékié par exemple, le GIC UGICCAMM créé en 2003 cultive le cacao. Le tableau 3 montre l'évolution de la production cacaoyère de ce GIC de 2011 à 2015.

Tableau 3. Evolution de la production cacaoyère du GIC UGICCAMM de 2011 à 2015

\begin{tabular}{c|c}
\hline Années & Production (en tonnes) \\
\hline 2011 & 147 \\
\hline 2012 & 331 \\
\hline 2013 & 456 \\
\hline 2014 & 672 \\
\hline 2015 & 676 \\
\hline
\end{tabular}

Source : Rapports d'activités du GIC UGICCAMM de 2011 à 2015 
Il ressort du tableau 3 que la production cacaoyère de ce GIC a évolué de façon constante de 2011 à 2015 . En effet, parti de $147 \mathrm{t}$ de cacao en 2011, la production a rapidement progressé pour atteindre 676 t en 2015, en raison de l'application rigoureuse des mesures de protection phytosanitaire, notamment la lutte contre la pourriture brune des cabosses de cacaoyers et la protection des plantations contre les capsides. ${ }^{20}$

En dehors de la production, les organisations paysannes ont également comme activités la transformation des produits agricoles.

\section{La transformation des produits agricoles}

Les activités de transformation des produits agricoles sont menées par des GICS qui disposent d'une certaine assise financière et des équipements adéquats. C'est le cas du GIC SOCOAPACE dans la Lékié qui dispose d'une unité de transformation du manioc. Elle comprend deux cossetteuses, un moulin à pâte, une risée, un moulin sec, un tamis motorisé, un four à séchage, un tamis et une poêle à gari acquise en 2015. Ce GIC dispose aussi d'un atomiseur, d'une motopome et d'un magasin de stockage. ${ }^{21}$

Dans le Centre, les produits transformés sont principalement le manioc, avec ses dérivés comme le gari, le couscous de manioc, les bâtons de manioc, l'amidon, la farine et le cacao dont on extrait le beurre très prisé sur le marché en raison de ses nombreuses vertus thérapeutiques, cosmétiques et diététiques. Il convient de noter que l'extraction du beurre de cacao se fait de façon artisanale. Le paysan fait griller les fèves de cacao, les écrase, puis met la pâte à ébullition et recueille l'excès d'huile. C'est le cas du GIC Khabia dont les membres sont spécialisés dans la fabrication du beurre de cacao. ${ }^{22}$ Mais cette technique d'extraction artisanale est critiquée par les fabricants de beurre de cacao pressé à froid. Ces derniers pensent que le beurre perd à travers la technique d'extraction artisanale une partie de sa valeur nutritionnelle.

D'autres produits font également l'objet d'une première transformation. C'est ainsi que depuis 1995, la fédération des GICS de la région d'Essé sèche les légumes en grande quantité qui sont ensuite écoulé dans plusieurs villes du Cameroun notamment Yaoundé, Mfou, Mbalmayo, Bafia et Soa. Depuis 1996, elle produit annuellement environ 1600t de farine de maïs et 2000t de sémoule de manioc, communément appelé gari. En 1993, l’Union des Groupements Communautaire d'Essé (UGCE) avait fabriqué environ 5000t de provende de maïs destinées aux éleveurs. En 1994, l'UGCE a reçu du Fonds Européen de Développement (FED) une bagatelle somme de 97000000 de francs CFA pour la production de la provende de maïs. ${ }^{23}$ Certains GICS comme le GIC Cel d'Elomzok dans la Lékié extrait l'huile de palme à partir des noix de palme. Le GIC Wamkok de la même localité transforme le soja en provende pour la nutrition de la volaille et des porcs. ${ }^{24}$

En dehors de la transformation de la production agricole, les organisations paysannes ont également comme activité la commercialisation. 


\section{La commercialisation des produits}

Une grande partie de la production agricole issue des organisations paysannes est destinée à la consommation. Toutefois, le surplus est écoulé dans les marchés, essentiellement dans les villes. Les GIC disposent des agents commerciaux qui ont pour rôle de vendre les produits des différents membres dans les villes où la demande est forte et où l'offre financière est alléchante. Ainsi, dès que les agents commerciaux rassemblent le surplus de la production, ils louent un véhicule pour transporter leurs marchandises dans les villes comme Yaoundé, Mbalmayo, Soa, Mfou et bien d'autres.

Certains agents se dirigent vers les villes plus éloignées comme Douala, Kribi, Ambam, Bitam, Oyem et même à Libreville au Gabon. Ces produits sont généralement livrés aux revendeurs sédentaires qui en assurent la distribution en détail aux consommateurs. C'est ainsi que par exemple en 2000, l'Union des groupements communautaire d'Essé a livré dans les villes $\mathrm{du}$ Cameroun près de $1300 \mathrm{t}$ de plantain, $230 \mathrm{t}$ de farine de mais et $370 \mathrm{t}$ de sémoule de manioc pour une valeur total de 49000000 de francs CFA. ${ }^{25}$

En 2001, l'Union des Groupements et d'Entraide de la Mefou et Akono (UGEMA) a vendu dans les villes de Yaoundé, Edéa, Douala, Ebolowa et Libreville environ $1550 \mathrm{t}$ de plantain, $485 \mathrm{t}$ de farine de maïs et $200 \mathrm{t}$ de semoule de manioc pour un montant de 75000000 de francs CFA. Après déduction des charges, des cotisations et de l'épargne commune, cet argent a été reparti entre les membres des GICS au prorata de la quantité des produits fournis par chacun d'eux. ${ }^{26}$

Pendant la campagne agricole 2011/2012, la Société Coopérative des Planteurs de Cacao et des Vivres de la Lékié a effectué la commercialisation groupée de cacao. Cette opération comprenait 24 ventes avec 3 acheteurs grossistes. Ainsi, $62 \mathrm{t}$ de cacao ont été vendu pendant cette période. ${ }^{27}$

En 2012, le GIC Cel d'Elombok a vendu dans les marchés du centre 124 filets de manions de $50 \mathrm{kgs}, 212$ filets de patate de $50 \mathrm{kgs}, 500$ régimes de banane douce et 300 régimes de banane plantain. ${ }^{28}$ En 2015, la SOCOAPCE COOP-A a commercialisé $25 \mathrm{t}$ de cacao, 3000 régimes de plantain, 10000 pieds de bananiers, 20000 boutures de manioc, 3,5 t de maïs, 20 bœufs, 150 porcs, 250 poulets, 1,5 t d'amidon, 200000 bâtons de manioc, $4 \mathrm{t}$ de farine et $500 \mathrm{kgs}$ de sémoule de manioc. ${ }^{29}$ Cette opération a permis au groupe d'engranger une rondelette somme de 37762500 FCFA. Il convient de préciser que la méthode de commercialisation la plus prisée est celle des ventes groupées dont les avantages sont la diminution des problèmes de transport, l'augmentation des marges bénéficiaires et l'imposition des prix de vente sur le marché. ${ }^{30}$

En dehors de la commercialisation, les regroupements paysans de la région du Centre ont comme autre activité l'épargne. 


\section{La promotion de l'épargne}

Les revenus d'un groupement procèdent des sources de recettes diverses qui comprennent les cotisations des membres, les dépôts volontaires effectués par les membres dans les caisses du groupement, les revenus des exploitations communautaires, le produit de la prestation de services, les dons et les prêts etc. A partir de ces sources de revenus, l'épargne réalisée par les groupements constitue le facteur décisif qui doit conditionner la réussite du processus de prise en charge par les producteurs des fonctions de productions. ${ }^{31}$

La cotisation est un système informel de thésaurisation de la monnaie. Les membres concernés collectent des fonds qui sont remis au bénéficiaire du jour suivant un ordre déterminé à l'avance. Ces cotisations sont plus courantes dans les tontines. C'est le cas de l'association "tout vient avec le temps". Les opérations menées par les tontines pour la collecte des fonds portent essentiellement sur les montants d'adhésion, la caisse de secours dont la contribution individuelle s'élève à $800 \mathrm{~F} /$ mois, la caisse d'épargne scolaire qui est volontaire.

Dans les associations, le mécanisme de caisse est mis en place pour permettre à chaque membre d'épargner et de faire fonctionner le groupe. Dans la Centrale Rurale de Développement Autogéré de Ngoazip (CERDAN) dans le Sud par exemple, le mécanisme de mobilisation des ressources financières porte sur la collecte des montants d'adhésion à raison de 1000 francs CFA pour les membres et 2000 francs CFA pour les groupes; les dépôts à vue qui sont rémunérés, les dépôts à terme d'une durée de 6 à 12 mois également rémunérés et bien d'autres. ${ }^{32}$ Le tableau 4 présente la situation financière du CERDAN de 2000 à 2002

Tableau 3. Situation financière du CERDAN de 2000 à 2002

\begin{tabular}{l|c|c|c|c}
\hline \multicolumn{1}{c|}{ Adhésions } & $\mathbf{2 0 0 0}$ & $\mathbf{2 0 0 1}$ & $\mathbf{2 0 0 2}$ & Cumul \\
\hline Nombre total & 100 & 161 & 171 & 171 \\
\hline Hommes & 70 & 108 & 112 & 112 \\
\hline Femmes & 29 & 52 & 57 & 57 \\
\hline Groupes & 01 & 01 & 02 & 02 \\
\hline Montant total & 104000 & 165000 & 179000 & 179000 \\
\hline Dépôts à vue & \multicolumn{5}{|l}{} \\
\hline Montant total & 111595 & 1268365 & 1029065 & 2409025 \\
\hline Hommes & 92845 & 838365 & 624020 & 1554900 \\
\hline Femmes & 12750 & 361030 & 364985 & 738765 \\
\hline Groupes & 69300 & 69300 & 40060 & 115360 \\
\hline
\end{tabular}




\begin{tabular}{l|l|l|l|l|l|l|l|l}
\hline Dépôts à terme \\
\hline & Nbre & Montant & Nbre & Montant & Nbre & Montant & Nbre & Montant \\
\hline $\begin{array}{l}\text { Mont a nt t } \\
\text { total }\end{array}$ & 19 & 97200 & 93 & 595785 & 38 & 502305 & 150 & 1195290 \\
\hline Hommes & 07 & 68500 & 21 & 169020 & 14 & 306025 & 42 & 543545 \\
\hline Femmes & 12 & 28700 & 72 & 426765 & 23 & 188280 & 107 & 643745 \\
\hline Groupes & & & & & 01 & 8000 & 01 & 8000 \\
\hline Dépôts actions & 60 & 390500 & 161 & 1870355 & 88 & 1575570 & 309 & 3836420 \\
\hline Hommes & 43 & 255000 & 127 & 1417355 & 70 & 1033010 & 24 & 2705365 \\
\hline Femmes & 16 & 100500 & 30 & 364400 & 18 & 542560 & 64 & 1007462 \\
\hline Groupes & 01 & 35000 & 04 & 88600 & & \multicolumn{5}{l}{0} \\
\hline
\end{tabular}

Source : Les données sont tirées des rapports d'INDAES Formation de 2000 à 2002

Il ressort du tableau 4 qu'à sa création, le CERDAN a connu une adhésion massive des paysans. Le nombre de membres a évolué progressivement passant de 100 adhérents en 2000 à 161 en 2001 et 171 en 2002. Cette adhésion massive est due au désir collectif d'épargner de la part des paysans et de la forte sensibilisation menée auprès d'eux par les membres du GIC. On constate aussi que le volume de l'épargne est considérable avec un montant total de 7440740 francs CFA épargné dont 2409025 francs CFA de dépôts à vue. ${ }^{33}$

Toutes les actions que nous venons de décrire ont eu une incidence heureuse sur la vie des populations membres des organisations paysannes.

\section{Impact socioéconomique de l'action des organisations paysannes}

Les activités menées par les membres des GIC contribuent, à n'en pas douter, à améliorer leurs conditions de vie. Des changements sont observables aussi bien sur les conditions de vie individuelle que sur les techniques agricoles.

\section{L'amélioration des conditions de vie des membres}

Les membres des organisations de producteurs tirent profit des diverses activités socioéconomique du groupe grâce aux bénéfices générés par la vente de leurs produits. Les différentes actions menées à savoir le développement de l'agriculture, l'élevage et le commerce sont en elles mêmes des stratégies de lutte contre la pauvreté. Le FIDA estime à cet égard que la réduction de la pauvreté n'est pas seulement la tâche de l'Etat, mais une prise de conscience 
des pauvres eux-mêmes de leur situation et qui deviennent des acteurs du changement. ${ }^{34}$

Dans cette perspective, les membres des GIC utilisent les bénéfices générés par leurs activités pour acheter les objets utiles dans la vie quotidienne comme des marmites, des assiettes, des meubles en bois ou les matériaux de construction comme les tôles, le ciment, les planches, les lattes, les clous et bien d'autres choses. ${ }^{35} \mathrm{Ce}$ qui a poussé les dirigeants des associations paysannes à procéder eux-mêmes aux achats est le fait que certains paysans n'utilisaient pas toujours à bon escient les sommes perçues. Pour résoudre ce problème, ils ont mis sur pied ce système qui impose à chaque membre d'indiquer à l'avance ce dont il a besoin comme meuble ou matériel pour améliorer son vécu quotidien. Cette façon de procéder a permis aux paysans membres des GICS de s'équiper. C'est par exemple le cas de Guy Roger Onana qui affirme avoir perçu entre 2009 et 2015 des matériaux de construction du GIC COOPRAEC. ${ }^{36}$ Ce matériel joint aux autres revenus lui ont permis de construire une maison.

Les activités agricoles permettent aussi aux membres des GIC d'améliorer leur alimentation en ce sens qu'une bonne partie des produits récoltés est destinée à l'autoconsommation. C'est le cas de Marthe Owona qui affirme détenir toujours un minimum de vivre dans son grenier. ${ }^{37}$

L'épargne effectué par les membres des organisations paysannes et qui est redistribuée entre le mois d'août et de septembre en fonction des intérêts et des investissements de chaque paysan leur permet de s'occuper de leur santé et de la scolarité de leurs enfants. En effet, pendant la rentrée scolaire, les parents utilisent cet argent pour s'occuper de la scolarité de leurs progénitures. Chantal Menyeng, membre du GIC Espoir de Ngouleman affirme avoir perçu 173000 francs CFA en septembre 2014, après la cassation de leur caisse ; ce qui lui a permis d'assurer le paiement des frais de scolarité de son fils. ${ }^{38}$ D'autres l'utilisent pour se soigner. C'est le cas par exemple de Lise Mballa du GIC CAVO qui a utilisé son épargne pour payer les frais médicaux de sa fille. ${ }^{39}$ Certains paysans utilisent leur épargne pour créer des projets comme l'élevage des porcs, des poulets ou même une boutique.

En dehors de l'amélioration des conditions de vie des membres, l'action des organisations paysannes a aussi entraîné des changements sur le plan socioculturel.

\section{Les changements culturels et économiques}

Selon Lavigne Delville, ${ }^{40}$ les organisations paysannes constituent, Sur le plan socioculturel, un cadre idéal pour la vulgarisation des innovations techniques en agriculture. Dans la Lékié par exemple, le Groupe d'Entraide Fraternel (GEF) constitue un cadre privilégié d'expérimentation et de réflexion sur le développement de la région. Les membres de ce GIC ont pu bénéficier des sessions de formations sur la culture des échalotes (petites gousses d'oignons) 
et leur mise en pratique dans les plantations. Les membres d'une autre organisation paysanne le GIC Apeyl, ont bénéficié de la formation organisée par Heifer International sur la fabrication du compost, les techniques de semi du maïs, l'entreprenariat agricole et la pépinière du cacao. ${ }^{41}$ Dans le Nyong et Mfoumou, le GIC Espoir a permis à ses membres d'acquérir des techniques de transformation des tubercules de manioc en cossettes et la vulgarisation de ces techniques se fait maintenant par les membres de cette association, qui est devenue prestataire de service auprès des autres groupes. ${ }^{42}$

Sur le plan économique, les organisations paysannes par leurs activités sont à la base de plusieurs changements économiques dont la modification du circuit économique. Ces organisations prennent généralement le relais pour la commercialisation primaire. C'est le cas par exemple du GIC Espoir de Ngouleman dans le Nyong et Mfoumou qui s'occupe de la filière manioc et de sa commercialisation à Mvog Mbi à Yaoundé. Ce GIC produit des bâtons de manioc et procède à la recherche des débouchés et partenaires.

\section{Conclusion}

En conclusion l'objet de cet article était d'analyser le rôle joué par les organisations paysannes dans la promotion du monde rural dans la zone forestière du Cameroun méridional. Il ressort des développements effectués que le désengagement de l'Etat du secteur productif ainsi que le cadre législatif et réglementaire mis en place dans les années 1990 ont favorisé l'émergence des regroupements paysans. Ceux-ci contribuent de diverses manières au développement rural notamment par les activités agricoles, la transformation et la commercialisation des produits de leurs membres et la mobilisation de l'épargne. Les revenus issus de ces activités permettent à leurs membres de résoudre leurs problèmes quotidiens et d'améliorer leurs conditions de vie.

\footnotetext{
Notes

* Université deYaoundé I. Yaoundé, Camarões. E-mail: signiefrs@yahoo.fr

1 Georges Courade . “Organisations paysannes, sociétés rurales, Etat et développement au Cameroun (1960-1980)." In : Actes du colloque sur l'économie politique du Cameroun, perspectives historiques. Juin 1988. pp.58.

2 Dogngmo, J.L. “ Les dynamismes endogènes et leur fondement culturel.”In : Simo David. La politique de développement à la croisée des chemins : le facteur culturel. Yaoundé : éditions clé, 1998. p.178-192.

3 P. Menye Atangana, "Regroupements paysans et développement socioéconomique dans la Lékié, 1992-2015”, Mémoire de DIPES II en histoire, Ecole Normale Supérieure de Yaoundé, 2017.

4 Ekoué Ekoué, E. "Contribution des organisations paysannes à la lutte contre la pauvreté.” Mémoire d'économie et de sociologie rurale. Université de Dschang, 2003.
} 
5 République du Cameroun. Stratégie de développement du secteur rural. p.4.

6 Fongang G. "Evolution et défis du mouvement paysan au Cameroun". Grain de sel n49, janvier à mars 2010, p.1.

7 Entretien avec François Seh, 53 ans, Sous directeur de la qualité totale et de l'appui aux Organisations paysannes à la SODECAO, Yaoundé, 17 Juillet 2016.

8 Mercoiret M.R., "Les organisations paysannes et les politiques agricoles." Afrique contemporaine, $\mathrm{n}^{\circ} 217$, janvier 2006, p.4.

9 Bila, S. "Analyse des stratégies paysannes de lutte contre la pauvreté par les organisations de producteurs dans la province du centre Cameroun." Mémoire d'ingénieur agronome, Université de Dschang, 2005, p.9.

10 Kambéza, S. "La structuration et le fonctionnement des organisations paysannes." FAO, 2007, p.4.

11 Kengne Fodouop. "Le développement rural dans la province du centre au Cameroun.," Les Cahiers d'outre-mer n'226-227, Avril-Septembre 2004, pp.89.

12 ibid

13 Ekoué, Ekoué. “Contribution des organisations”, p.12.

14 Ibid., p.13.

15 Entretien avec Elise Béyala, 43 ans, présidente de la coopérative agricole de Khabia, Batshenga, 02 juin 2017.

16 ibid

17 Rapport d'activités du comité de développement de la vallée de Kya

18 Rapport d'activités du GIC MAT, 1993.

19 Rapport d'activités du GIC Cel d'Elombok, 2012.

20 Entretien avec Richard Atangana, 48 ans, cacaoculteur, Nkolafamba, 15 juin 2017.

21 Ménye Atangana. "Regroupements paysans", p.62.

22 Entretien avec Angèle Alima, 47 ans, gestionnaire de la ferme agricole de Missolé, Yaoundé, 5 juin 2017.

23 Kengne Fodouop. "Développement rural”, p.89.

24 Entretien avec Justin Touong, 42 ans, conseiller de groupement des producteurs ACEFA, Okola, 8 juin 2017.

25 Rapport d'activités du GIC UGEMA, 2002, p.2.

26 Kengne Fodouop, "Développement rural”, p.90.

27 Rapport d'activités 2012 de la SOCOOPLACAB.

28 Rapport d'activités du GIC CEL, 2012

29 Rapport d'activités de la SOCOOPMACE-COOP-A 2015.

30 Entretien avec Yannick Onana, 34 ans, chef de la cellule de statistique à la délégation départementale de l'Agriculture de la Lékié, Yaoundé, 8 juin 2017.

31 Archives de la SODECAO, Enquêtes d'évaluation de la capacité d'épargne au sein des groupements de producteurs, p.2. 
Revista Esboços, Florianópolis, v. 25, n. 39, p. I31-146, jul. 2018.

$32^{32}$ Rapport d'activités INADES Formation

$33^{33}$ Rapports d'activités d'INADES-Formation 2002.

$34^{34}$ Bila, s. Analyse des stratégies de lutte contre la pauvreté, p.45

$35^{35}$ Entretien avec Gisèle Noa, 50 ans, Paysanne, membre du GIC Apeyl, Okola, 10 juin 2017

$36^{37}$ Entretien avec Guy Roger Onana, 46 ans, membre du GIC COOPRAEC, Okola, 10 juin 2017

$37^{38}$ Entretien avec Marthe Owona, paysanne, Yaoundé, 08 juin 2017.

$38^{39}$ Entretien avec Chantal Menyeng, 43 ans, membre de GIC, Yaoundé, 11 juin 2017.

39 Entretien avec Lise Mballa, 43 ans, membre du GIC CAVO, Okola, 10 juin 2017

$40^{40}$ Lavigne Delville. Les Associations paysannes en Afrique : organisation et dynamique. Paris : Karthala, 1986. p.32.

$41^{41}$ Rapport d'activité du GIC APEYL, 2011, p.3.

$42^{42}$ Ekoué Ekoué. "Contribution des organisations", p.38.

Recebido em: 20/12/2017

Aprovado em: 17/02/2018 